\title{
2006-2649: IMPROVE LEARNING EFFICIENCY WITH INTEGRATED MATH AND CIRCUIT SIMULATION TOOLS IN ELECTRICAL AND COMPUTER ENGINEERING COURSES
}

\section{Colin Campbell, TechnicalMastery.com Corp.}

Colin Campbell works with professors in Engineering at the University of Waterloo assisting them integrate mathematical software into their courses for the purpose of design and simulation.

Colin graduated from Waterloo's "Co-op Applied Math with Engineering Electives" programme in 1982.

Colin is the owner of TechnicalMastery.com Corporation which develops on-line video-based training courses, with an engineering leaning, for mathematical software such as MATLAB, Mathcad and Maple.

\section{Faycal Saffih, McMaster University}

Fayçal Saffih was born in 1974, in Sétif, Algeria. He has got his B.Sc. with best Honors in Solid-State Physics from Physics Department, University of Ferhat Abbass, Sétif, Algeria in 1996. He pursued his studies on artificial neural networks and achieved his master degree from Physics Department, University of Malaya, Malaysia in 1998 for his research on "Digital Implementations of Artificial Neural Networks". He joined in 1999 the Integrated Camera Group at the Electrical and Computer Engineering Department, University of Waterloo, Ontario, Canada, and achieved his Ph.D. on "Foveated Sampling Architectures on CMOS Image Sensors" dedicated for video communication and remote imaging. Currently, he is a Visiting Scholar to the Communication Research Laboratory, McMaster University in Hamilton, Ontario, Canada, working on research and development of "Smart Imaging Systems for Biomedical Applications" such the Endoscopic Capsule.

\section{Khaled Nigim, University of Waterloo}

Khaled Nigim is a registered professional engineer in Ontario, Canada, senior member of the IEEE, has a Ph.D. in Electrical Engineering from the University of Leicester, England UK and a B.Sc. in Electrical Engineering from Zagazig University of Cairo, Egypt. He is currently the coordinator of the Master of Engineering professional development graduate program offered on-line at the University of Waterloo, E\&CE department. 


\section{Improve Learning Efficiency with Integrated Math and Circuit Simulation Tools in Electrical and Computer Engineering Courses}

\section{Abstract}

This paper presents coupling the use of the TINA circuit simulation software with the Mathcad mathematical software. This coupling permits students to simply (1) enter a circuit in TINA diagramatically, (2) export its symbolic solution $y(t)$, or its transfer function, $\mathrm{Y}(\mathrm{s})$, to a Mathcad file, and (3) plot these solutions for multiple values of a parameter (e.g. R) on a 2-D or 3-D graph. The symbolic solutions and plots enhance understanding of both the physical and the mathematical foundations of the studied cases. We envision this coupling being used in classrooms by instructors, and by students. (This coupling only works in the case of linear circuits, so for example it does not work with diodes).

\section{Introduction}

In our first example, we enter an RLC circuit into TINA with fixed values of R, L and C. Then we find the symbolic solution in terms of unknown R, $\mathrm{L}$ and $\mathrm{C}$. Finally we export the symbolic solution to Mathcad where we plot I $(t, R)$ as a surface plot varying $t$ and $R$. From the plot and the symbolic solution we make observations about the behavior of the solution as $R$ varies - which are valuable educationally and from a design perspective.

In our second example, we enter a low-pass filter into TINA with $\mathrm{C}_{\text {in }}$ as the parameter of interest. Then we find the transfer function symbolically in terms of $\mathrm{C}_{\text {in. }}$. Next we export the transfer function $\mathrm{W}\left(\mathrm{s}, \mathrm{C}_{\mathrm{in}}\right)$ to Mathcad where we create Bode plots with various values of $\mathrm{C}_{\text {in }}$ all on the same axes for comparison. From the plot we make observations about the behaviour of the filter as $C_{\text {in }}$ varies. Finally we get Mathcad to take the Inverse Laplace Transform of W(s, Cin)/s to get the step response, $y\left(t, C_{i n}\right)$, which we plot with various $C_{i n}$ values, and make further observations.

\section{TINA}

TINA is comprehensive circuit simulation software made by DesignSoft, Inc. What sets it apart from other such software, among other things, is its ability to produce symbolic solutions to circuits [1], and to produce symbolic transfer functions, both of which are very useful in educational settings. (www.TINA.com)

\section{Mathcad}

Mathcad is comprehensive mathematical software made by MathSoft, Inc. What sets it apart is that mathematics are entered and displayed in familiar math notation [2, 3], unlike a textual programming language. Whenever you change a variable, Mathcad recomputes any variables that depend on it, making it very useful in education and design. (www.Mathcad.com) 


\section{Example 1 - RLC Circuit}

Consider the following RLC circuit created in TINA. This time we are measuring current:

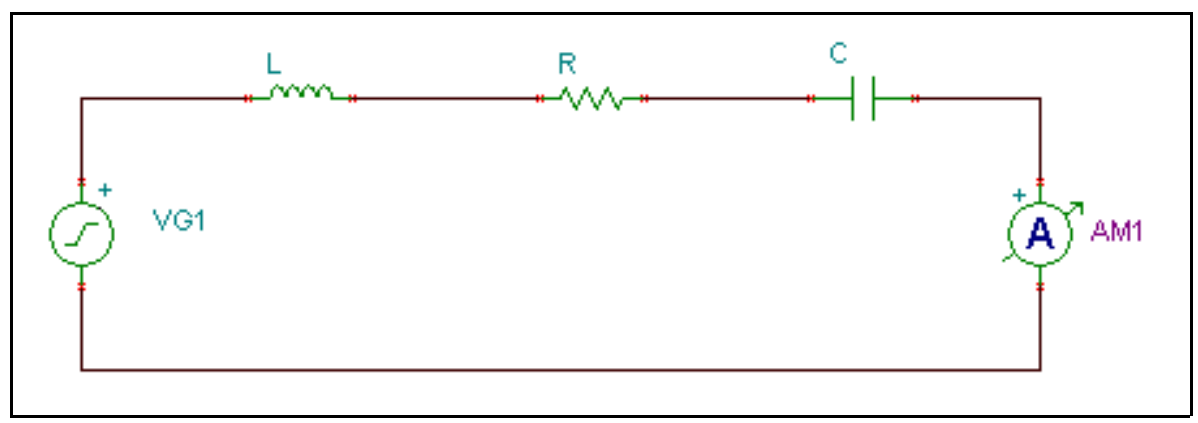

We asked TINA for the symbolic transient response, and we saved it to a Mathcad file. After a little editing, and turning $\boldsymbol{i}(\boldsymbol{t})$ into $\boldsymbol{i}(\boldsymbol{t}, \boldsymbol{R})$, the file now resembles:

$$
\begin{aligned}
& L:=0.001 \quad R:=1 \quad C:=0.001 \\
& A:=1 \quad \omega:=800 \quad \phi:=-90 \quad \operatorname{deg}:=\frac{2 \cdot \pi}{360} \\
& i(t, R):=A \cdot\left|\frac{C \cdot(j \cdot \omega)}{1+C \cdot R \cdot(j \cdot \omega)+C \cdot L \cdot(j \cdot \omega)^{2}}\right| \cdot \cos \left[\omega \cdot t+\operatorname{deg} \cdot(\phi)+\arg \left[\frac{C \cdot(j \cdot \omega)}{1+C \cdot R \cdot(j \cdot \omega)+C \cdot L \cdot(j \cdot \omega)^{2}}\right]\right]
\end{aligned}
$$

$\boldsymbol{i}(\boldsymbol{t}, \boldsymbol{R})$ can be displayed with numerical coefficients. Notice it displays as a sine function now:

$$
\left.i(t, R):=i(t, R) \text { float }, 5 \rightarrow \frac{.800}{\left(.12960+.64000 \cdot R^{2}\right)^{\frac{1}{2}} \cdot \sin \left(800 \cdot t+\arg \left(\frac{\mathrm{i}}{.36000+.800 \cdot \mathrm{i} \cdot R}\right)\right)}\right)
$$

Let's plot $\boldsymbol{i}(\boldsymbol{t}, \boldsymbol{R})$ for various values of $\boldsymbol{R}$ :

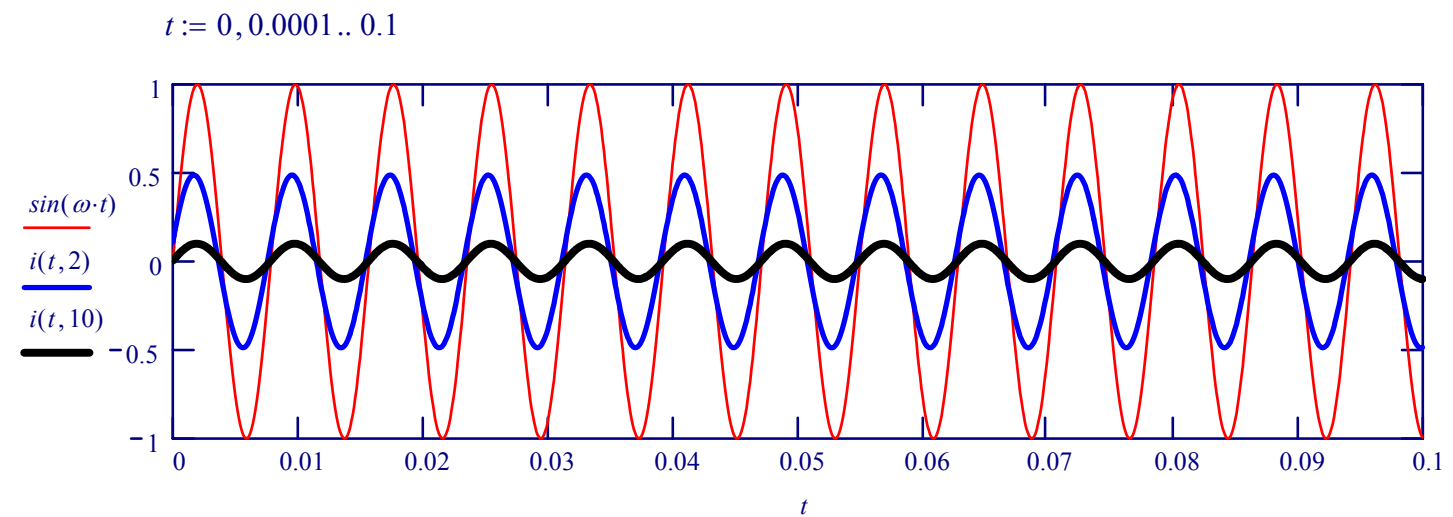




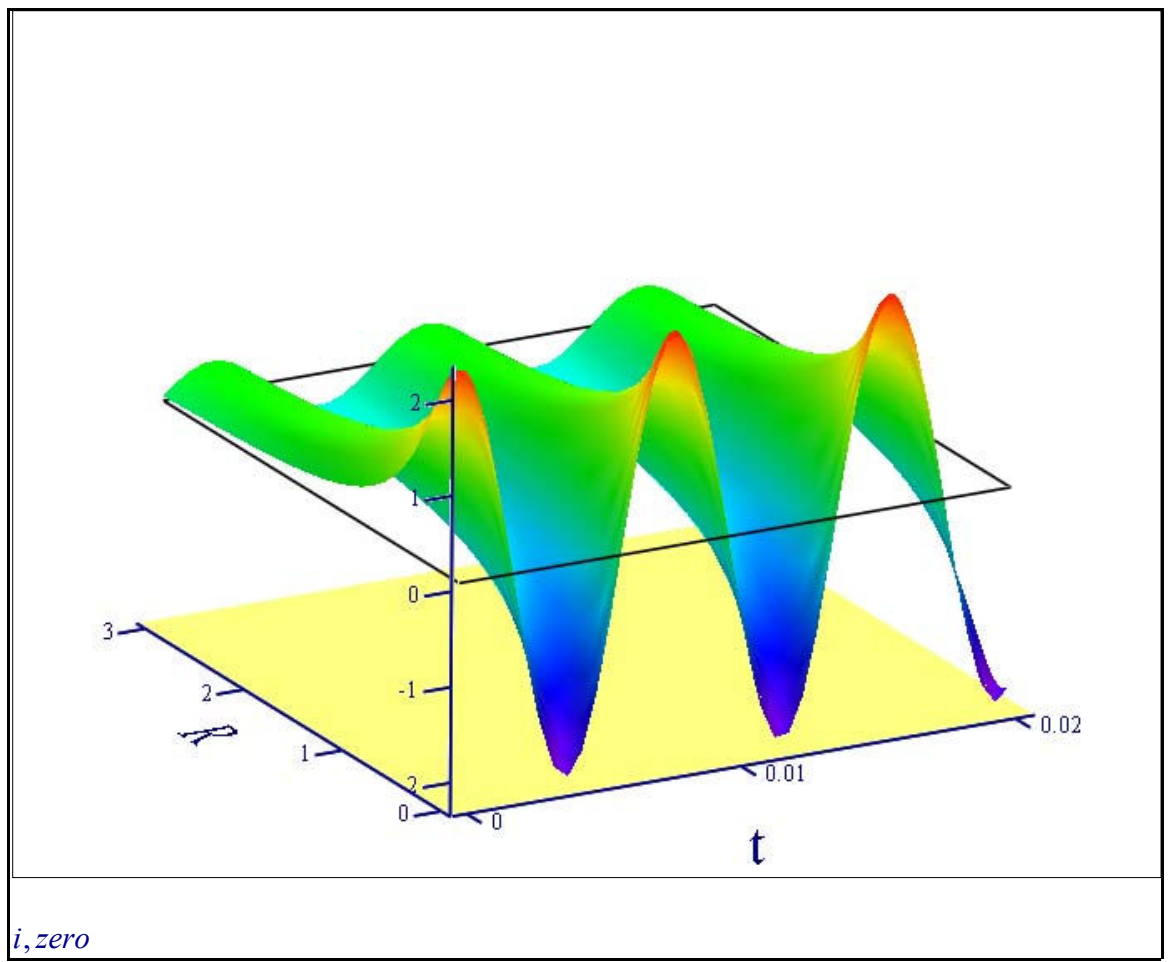

It is clear from this graph that as $\boldsymbol{R}$ increases, the response, $\boldsymbol{i}(\boldsymbol{t}, \boldsymbol{R})$ tapers off. And from our symbolic expression for the response, it is clear that $\boldsymbol{i}(\boldsymbol{t}, \boldsymbol{R})$ tapers off as $1 / \boldsymbol{R}$ (approximately).

\section{Example 2 - Low Pass Filter using Bipolar Transistor Amplifiers}

Consider the following low-pass filter circuit in TINA:

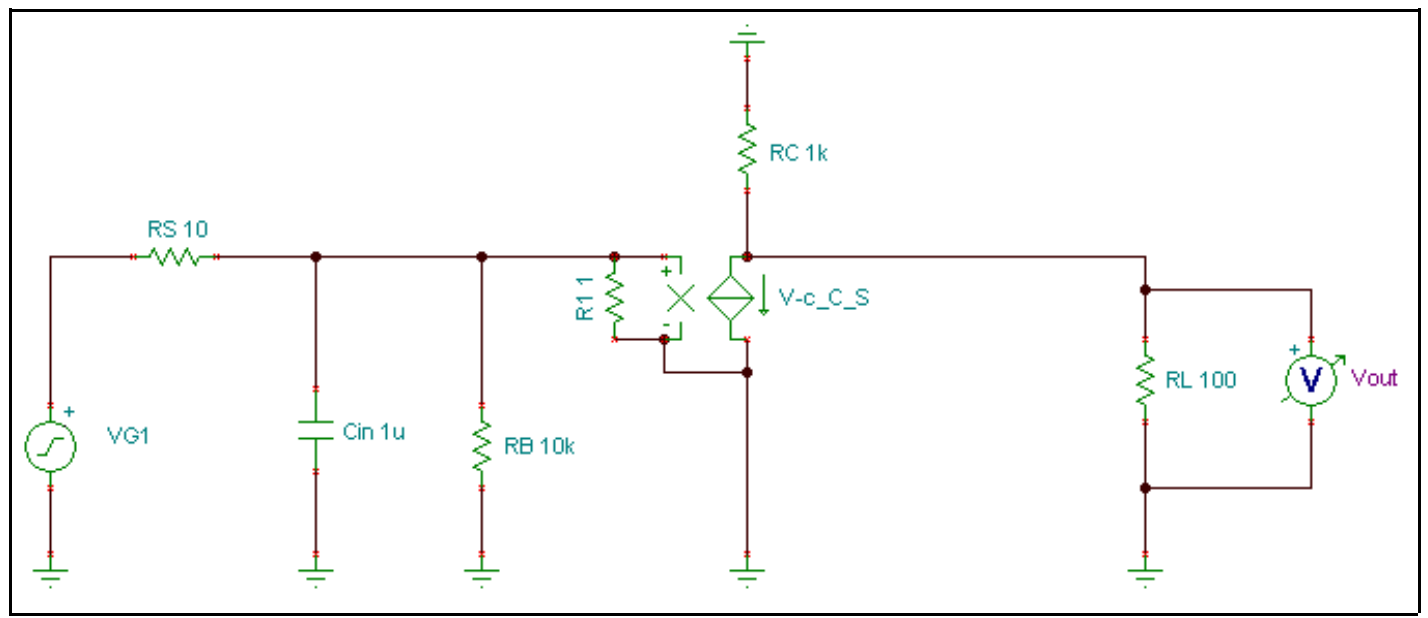


We asked TINA to produce the symbolic transfer function, and save it as a Mathcad file. This looks like the following, after a little rearranging and changing $\boldsymbol{W}(\mathbf{s})$ to be a function of $\boldsymbol{C i n}$ as well, i.e. $\boldsymbol{W}(\mathbf{s}, \mathrm{Cin})$ :

$$
\begin{aligned}
& \text { Cin }:=1 \cdot 10^{-6} \quad V:=1 \\
& R S:=10 \quad R B:=1 \cdot 10^{4} \quad R_{1}:=1 \quad R C:=1000 \quad R L:=100 \\
& V G I d c:=0 \quad V G I C:=6.123 \cdot 10^{-17} \quad V G I A:=1 \quad \text { VGIf }:=60 \quad V G l w:=377 \quad \text { VGIP }:=-90 \\
& W(s, C i n):=\frac{R_{1} \cdot V \cdot R L \cdot R C \cdot R B}{-R C \cdot R B \cdot R S-R L \cdot R B \cdot R S-R_{1} \cdot R C \cdot R S-R_{1} \cdot R L \cdot R S-R_{1} \cdot R C \cdot R B-R_{1} \cdot R L \cdot R B+(-R C-R L) \cdot C i n \cdot R_{1} \cdot R B \cdot R S \cdot s}
\end{aligned}
$$

We observe that as $\boldsymbol{C i n}$ increases, the magnitude of $\boldsymbol{W}(\mathbf{s}, \boldsymbol{C i n})$ decreases for a given value of $\boldsymbol{s}$. Let's create a Bode plot of $\boldsymbol{W}(\mathbf{s}, \boldsymbol{C i n})$ for various values of $\boldsymbol{C i n}$ on the same axes. This is very easily accomplished now that we have a symbolic expression for $\boldsymbol{W}(\boldsymbol{s}, \boldsymbol{C i n})$ :

$$
\begin{aligned}
& n:=1000 \quad i:=1 . . n \\
& \omega:=\operatorname{logspace}\left(10^{-2}, 10^{9}, n\right)
\end{aligned}
$$

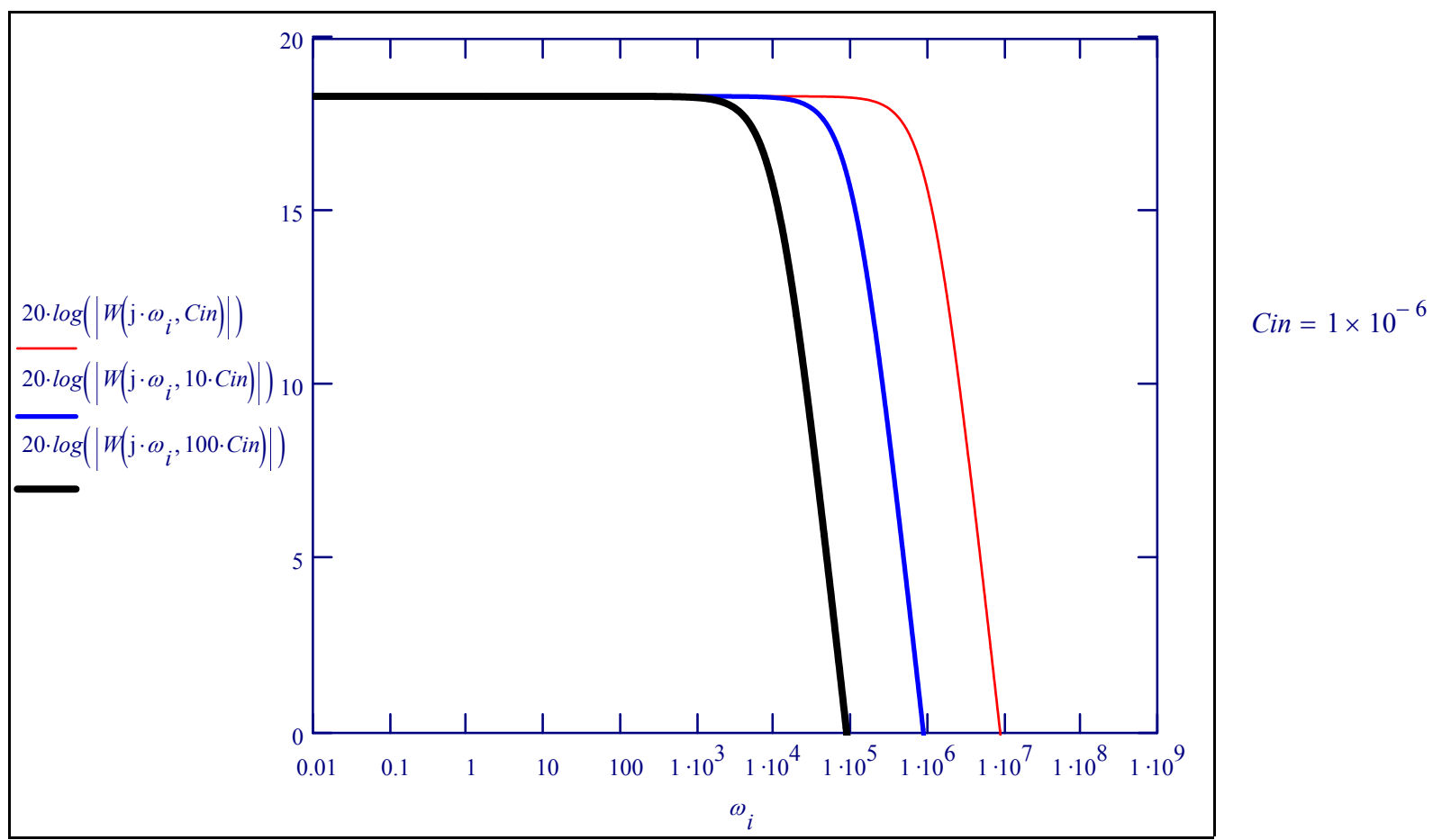

From the plot we see that as $\boldsymbol{C i n}$ is increases, the lowpass filter covers a smaller range of frequencies. 
Now let's consider the step response of the circuit by switching to the time domain. To do this we use the built-in invlaplace function in Mathcad to take the Inverse Laplace Transform of: $\boldsymbol{W}(\mathbf{s}, \mathrm{Cin}) / \mathrm{s}$, i.e.:

$$
y(t, \text { Cin }):=\frac{W(s, \text { Cin })}{s} \mid \begin{aligned}
& \text { invlaplace, } s, t \\
& \text { float }, 5
\end{aligned} \rightarrow \text { 8.2637.e } e^{-1.1001 \cdot \frac{t}{\text { Cin }}-8.2637}
$$

We notice that as $\boldsymbol{C i n}$ increases in the above definition, the more slowly that $\boldsymbol{y}(\boldsymbol{t}, \boldsymbol{C i n})$ changes. This can be seen in the following plot where $y(t, 10 C i n)$ changes more slowly than $y(t, C i n)$.

$$
t:=0,10^{-6} . .10^{-4}
$$

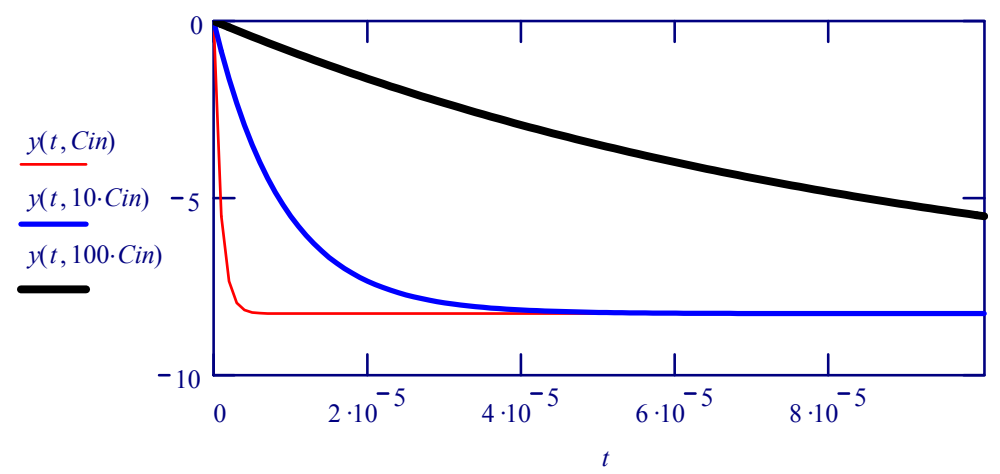

\section{Conclusions}

We have shown two examples of circuits that are typically found in the Electrical and Computer Engineering curriculum, that are easily entered graphically into a circuit analysis program like TINA. However, as we have seen, what sets TINA apart from other programs is its ability to compute symbolic expressions for (1) the transient solution of the circuit, and also (2) its transfer function - and export these to Mathcad for further work.

We have seen how the symbolic expressions give tremendous insight into how the various components in a circuit relate to one another. Furthermore, using Mathcad's 2-D and 3-D plotting abilities we are able to visualize on one plot the effects of varying any of the circuit's parameters. This shows the student the nature of the physical behaviour of the system in response to changes in the parameters, which is extremely valuable for learning and design.

Other advantages of this coupled approach to symbolic circuit analysis are:

1. Students work with circuits in familiar diagramatic form.

2. Students work with symbolic expressions in familiar mathematical form.

3. Problems are solved interactively, which increases student interest.

4. Students quickly get to a point where they can start making keen observations. 


\section{References}

1. Cooper, R. "The route to simulation III" [Review of TINA]. Electronics World, September 1999.

2. Domnisoru, C., "Using MATHCAD in Teaching Power Engineering". IEEE Transactions on Education, Vol. 48, No.1, February 2005.

3. Karady, G. and Nigim K., "Improve Learning Efficiency by Using General-Purpose Mathematics Software in Power Engineering". IEEE Power Engineering Education Journal, Vol. 18, No. 3, August 2003. 